\title{
Time-domain analysis of measurements on scaled urban models with comparisons to ray-tracing propagation simulation.
}

\author{
Danilo Erricolo Member, IEEE, Umberto G. Crovella Student Member, IEEE, \\ Piergiorgio L. E. Uslenghi Fellow, IEEE
}

\begin{abstract}
This work deals with propagation prediction for wireless communications in urban environments. Its purpose is to clearly distinguish the different field contributions collected by the receiving antenna through a series of wideband frequency measurements and a time domain analysis of the obtained results. The experimental data are then compared to the field predictions given by a ray-tracing electromagnetic wave propagation simulator. The application of this research lies in the modeling of the urban channel for wireless communication systems of the third generation (UMTS) and beyond.
\end{abstract}

Keywords - Anechoic Chamber, Propagation Predictions, Time-Domain Analysis, Urban Environment, UMTS, RayTracing, Geometrical Theory of Diffraction.

\section{INTRODUCTION}

$\mathrm{I}_{\mathrm{t}}^{\mathrm{N}}$ this paper a time-domain (TD) analysis is carried out to compare measurements with theoretical predictions about propagation in urban environments. The purpose of the TD analysis is to identify the multipath components that actually contribute towards the received field in a selected frequency band. The novelty of this study is that the measurements are conducted inside an anechoic chamber facility, using scaled models of urban environments and appropriate antennas. The advantage of this approach is that all parameters such as physical dimensions of the model and material properties are known with very low tolerances. Therefore, it is possible to make extremely accurate comparisons with the theoretical prediction obtained using a ray-tracing method.

In this paper, the experimental results are compared to the predictions given by the Polygonal Line (PL) simulator, which was described in [1], [2], [3] and compared with other methods in [4]. The results presented here complement those given in [5]. More specifically, the latter is about path-loss analysis at a single frequency, whereas this paper deals with the identification of the contributions from different trajectories by means of wideband measurements and a TD analysis. Since the two studies share the same experimental setup, the reader is referred to [5] for these details. In both papers, great emphasis is given to experiments that closely approach the simplified model of the real world that is analyzed with the theoretical method. In fact, the assumptions of the theoretical method are verified

The authors are with the Department of Electrical and Computer Engineering, University of Illinois at Chicago, 851 South Morgan St., Chicago, Illinois 60607, USA. E-mail: derricol@ece.uic.edu. This research was supported by NSF grant ECS 9979413. using the results of these experiments, an operation that is impossible to carry out with field measurements because too many parameters are unknown.

Nevertheless, this approach is not intended to substitute for field measurements, which are still very important, but simply to be used as one of the benchmarks to test new computer simulators and validate propagation models [6].

As it will become clear in the following sections, the close agreement between the predictions and the measurements validates the method of the PL simulator. Preliminary results of this investigation were given in [7], [8], [9], [10].

\section{Time-Domain Analysis}

The purpose of the TD analysis is the measurement and identification of the multipath components that actually contribute towards the received field in a selected frequency band. This requires the ability to resolve two closely time spaced multipath components, which we will refer to as response resolution, and the ability to locate the peak, in time, of a single multipath component, which we will refer to as range resolution [11].

The experiments consist of pulses that are launched from $T_{x}$ and measured at $R_{x}$. The pulses are generated by a Hewlett-Packard HP8510 Network Analyzer (NA) that operates in the frequency domain. The frequency bandwidth of operation determined by the radiation characteristics of the antennas is in the range from $19 \mathrm{GHz}$ to $27 \mathrm{GHz}$. Within this frequency range interval of $8 \mathrm{GHz}$, the NA considers 801 equally spaced samples, which results in a time period of $100 \mathrm{~ns}$ for the measured waveform. With 801 sample points, the response resolution of the system is $0.25 \mathrm{~ns}$ and the range resolution is $0.0625 \mathrm{~ns}$.

For a given scaled model of an urban environment, different configurations are considered. Each configuration is characterized by the positions of the transmitter and receiver with respect to the scaled building model. Given a certain configuration in input, the PL simulator computes the complex value of the EM field for a pre-determined set of frequencies. Both the experiments and the theoretical predictions return results in the frequency domain. The TD analysis was accomplished via post-processing using MATLAB, in order to guarantee full control and identical processing of the two sets of results. For both the measured and the simulated data, the sampling bandwidth in the frequency domain was extended by adding zero samples. The effect of adding zeroes corresponds to creating new samples 


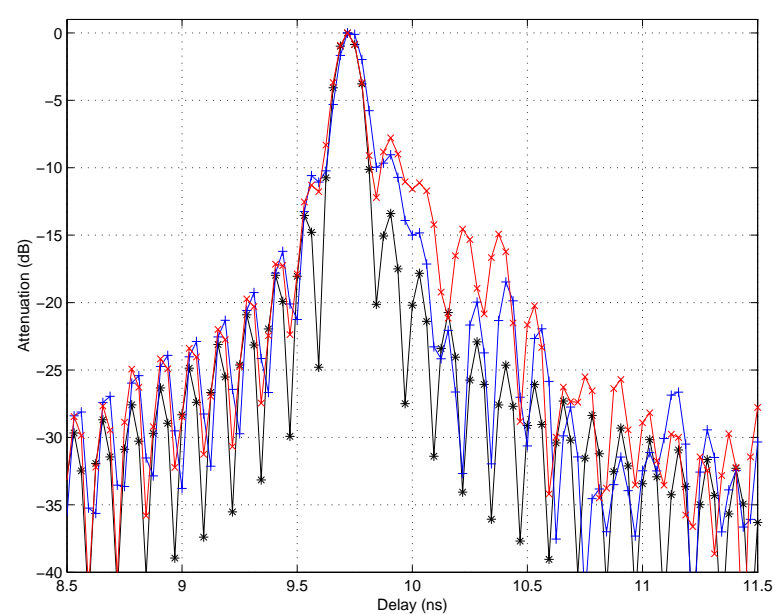

Fig. 1. Time-domain analysis for free space propagation. The curves show the PL simulator prediction (black line), and the measurements for soft (red line) and hard (blue line) polarization, respectively.

that interpolate the TD shape of the pulse. As a result, the pulse shape is less abrupt. This enlargement was limited to $32 \mathrm{GHz}$, i.e. four times the original frequency interval, in order not to corrupt the measurements too much. Additional details are found in [12].

The measured TD response for the case of free space propagation, after interpolation and normalization, is shown in Figure 1 for hard and soft polarization. The black line represents the impulse used for reference. The mean delay and attenuation of the main peak for this case are also summarized in Table I.

\section{TABLE I}

Mean Delay and ATtenuation of THE MAIN PEAK

\begin{tabular}{|c|c|c|}
\hline Polarization & Delay (ns) & Attenuation $(\mathrm{dB})$ \\
\hline Vertical (Hard) & $9.72( \pm 0.0625)$ & $-26.15( \pm 0.2)$ \\
Horizontal (Soft) & $9.8( \pm 0.0625)$ & $-27.2( \pm 0.4)$ \\
\hline
\end{tabular}

\section{Single-Building Profile}

To verify the accuracy of the PL simulator against the measured data in the case of propagation past buildings, let us start with the simple structure of the single building profile for different significant configurations. For all comparisons, the TD analysis is reported in graphs where the black line represents the PL simulator prediction and the measurement data are represented by red and blue lines for soft and hard polarization, respectively. In addition, the details of each multipath component are presented in a series of tables.

\section{A. Transmitter and receiver below rooftop and at different heights}

Let us consider the configuration shown in Figure 2 for the case of soft polarization. In this configuration the $R_{x}$ is only a few wavelengths from the ground. For this and

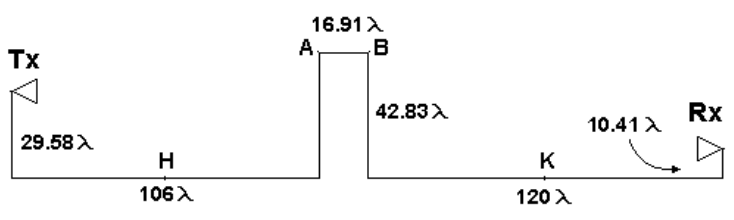

Fig. 2. Configuration 1: $T_{x}$ and $R_{x}$ below rooftop.

the single-building configuration that follows, the possible trajectories predicted by the PL are:

1. $T_{x} \rightarrow A \rightarrow B \rightarrow R_{x}$

2. $T_{x} \rightarrow A \rightarrow B \rightarrow K \rightarrow R_{x}$

3. $T_{x} \rightarrow H \rightarrow A \rightarrow B \rightarrow R_{x}$

4. $T_{x} \rightarrow H \rightarrow A \rightarrow B \rightarrow K \rightarrow R_{x}$

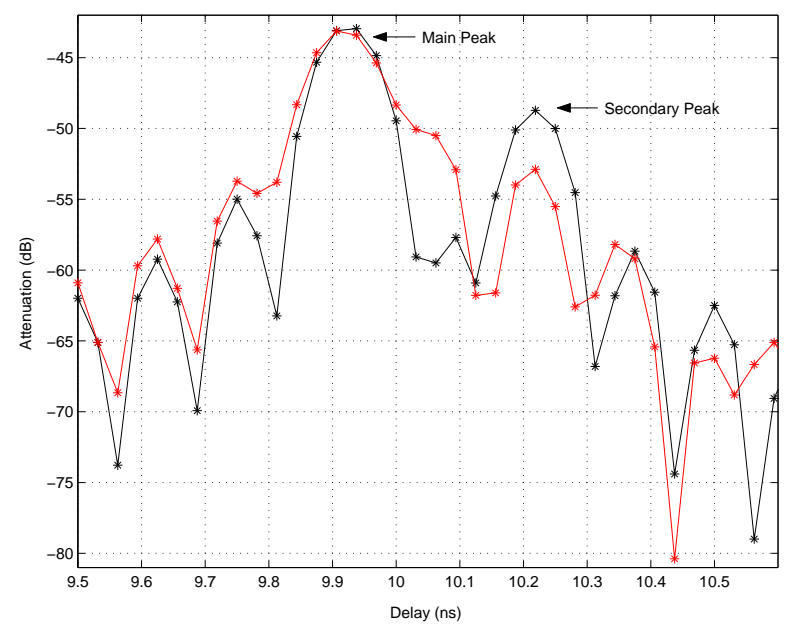

Fig. 3. Configuration 1: Time-domain analysis for soft polarization. The curves show the PL simulator prediction (black line) and the measurements (red line).

The TD analysis for this configuration is shown in Figure 3, which exhibits two peaks. The trajectories responsible for these peaks are easily recognized referring to Table II. In this configuration, the main peak is due to the double diffracted trajectory 1 , and the secondary peak to trajectory 2 . Notice that, since $R_{x}$ is only a few wavelengths above the ground plane, the paths $B \rightarrow R_{x}$ and $B \rightarrow K \rightarrow R_{x}$ provide similar contributions. The peaks associated with trajectories 3 and 4 are too weak to be

TABLE II

Configuration 1: Comparison Between measured data And PREDICTIONS GIVEN BY THE PL SIMULATOR

\begin{tabular}{|c|cc|cc|}
\hline & Measured & Data & PL & Simul \\
Trj & Delay $(\mathrm{ns})$ & Attn $(\mathrm{dB})$ & Delay $(\mathrm{ns})$ & Attn $(\mathrm{dB})$ \\
\hline 1 & 9.920 & -43.01 & 9.929 & -42.87 \\
2 & 10.220 & -52.87 & 10.208 & -50.88 \\
3 & $\mathrm{x}$ & $\mathrm{x}$ & 10.791 & -65.88 \\
4 & $\mathrm{x}$ & $\mathrm{x}$ & 11.071 & -73.33 \\
\hline
\end{tabular}


detected and they are immersed in the background noise.

\section{B. Transmitter and receiver at grazing aspects of incidence} and observation

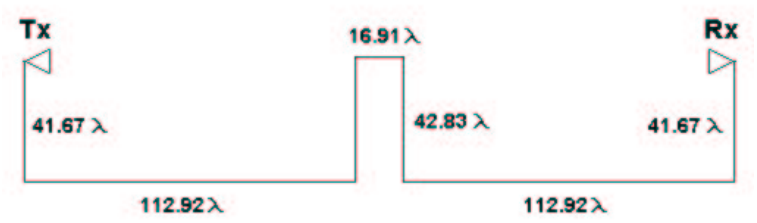

Fig. 4. Configuration 2: $T_{x}$ and $R_{x}$ at grazing incidence.

The configuration shown in Figure 4 is of particular importance because it challenges the ray-tracing algorithms by containing two transition zones. Also, it has the characteristic of being perfectly symmetrical. The experiments were carried out by actually keeping both the transmitter and the receiver slightly below the building rooftop to avoid direct ray contributions. The trajectories are the same indicated in Section III-A.

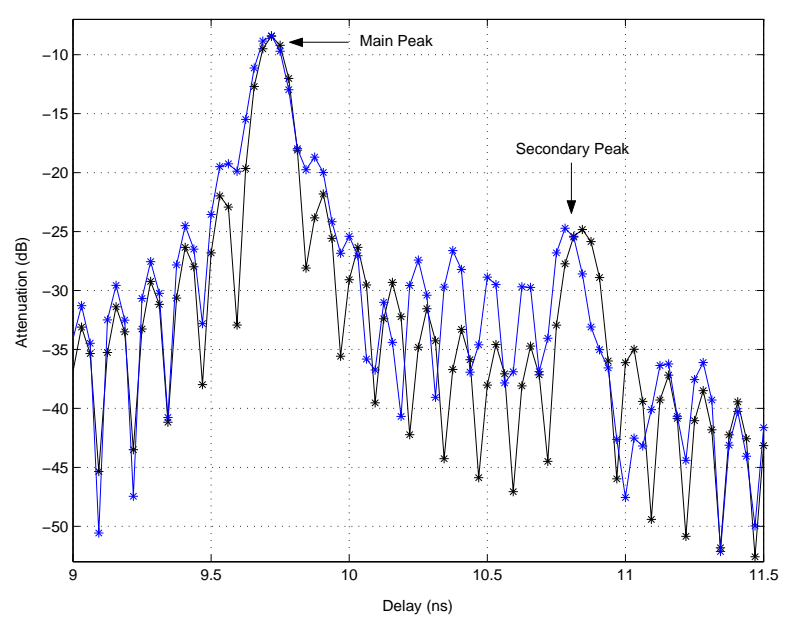

Fig. 5. Configuration 2: Time-domain analysis for hard polarization. The curves show the PL simulator prediction (black line), and the measurements (blue line).

The TD analysis for this configuration is shown in Figure 5. Taking into consideration the data of Table III, the main peak is associated with the double-diffracted trajectory 1 , whereas the secondary peak is due to a combination of both trajectories 2 and 3. In fact, because of the symmetry of the present configuration, trajectories 2 and 3 have equal path-lengths and their contributions are not separable.

When dealing with propagation over buildings, most of the ray tracing models used for wireless network planning consider only $R_{x}$-side ground reflection contributions, discarding the $T_{x}$-side ones. This choice is acceptable as long as the $T_{x}$ is located at a much higher height with respect to the $R_{x}$, otherwise it leads to a weaker prediction of the received field.
TABLE III

Configuration 2: Comparison Between measured DATA AND PREDICTIONS GIVEN BY THE PL SIMULATOR.

\begin{tabular}{|c|cc|cc|}
\hline & Measured & Data & PL & Simul \\
Trj & Delay (ns) & Attn $(\mathrm{dB})$ & Delay (ns) & Attn $(\mathrm{dB})$ \\
\hline 1 & 9.720 & -8.40 & 9.724 & -8.42 \\
2 & 10.795 & -24.72 & 10.839 & -29.60 \\
3 & $"$ & $"$ & 10.839 & -31.99 \\
4 & $\mathrm{x}$ & $\mathrm{x}$ & 11.953 & -48.43 \\
\hline
\end{tabular}

\section{Two-Building Profile}

The TD analysis of the multipath components is now applied to the case of the two-building profile. This profile represents the simplest case of rows of parallel buildings. In addition, since the two buildings have exactly the same height, it provides a way to challenge the ray-tracing algorithm because of the intrinsic grazing incidence condition created by this particular shape.

\section{A. Transmitter at grazing incidence}

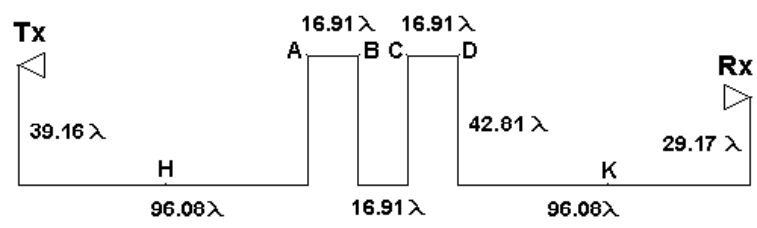

Fig. 6. Configuration 3: $T_{x}$ at grazing incidence.

Figure 6 shows the geometry for the configuration of the $T_{x}$ at grazing incidence. For this configuration, the trajectories that provide the most important contributions are:

1. $T_{x} \rightarrow A \rightarrow B \rightarrow C \rightarrow D \rightarrow R_{x}$

2. $T_{x} \rightarrow A \rightarrow B \rightarrow C \rightarrow D \rightarrow K \rightarrow R_{x}$

3. $T_{x} \rightarrow H \rightarrow A \rightarrow B \rightarrow C \rightarrow D \rightarrow R_{x}$

The TD analysis is shown in Figure 7, which corresponds to a hard-polarization case. Hard polarization was chosen because it results in stronger values for the received field and therefore makes the measurements easier.

The main peak of Figure 7 is easily identified with trajectory 1 of Table IV. The agreement with the theoretical prediction is very good both for the main and the secondary peaks. Also, notice that trajectories 2 and 3 provide similar contributions.

TABLE IV

Configuration 3: Comparison Between measured Data AND PREDICTIONS GIVEN BY THE PL SIMULATOR.

\begin{tabular}{|c|cc|cc|}
\hline Trj & $\begin{array}{c}\text { Measured } \\
\text { Delay (ns) }\end{array}$ & $\begin{array}{c}\text { Data } \\
\text { Attn }(\mathrm{dB})\end{array}$ & $\begin{array}{c}\text { PL } \\
\text { Delay }(\mathrm{ns})\end{array}$ & $\begin{array}{c}\text { Simul } \\
\text { Attn }(\mathrm{dB})\end{array}$ \\
\hline 1 & 9.750 & -21.25 & 9.763 & -21.36 \\
2 & 10.655 & -35.40 & 10.680 & -36.84 \\
3 & 10.934 & -38.65 & 10.964 & -41.07 \\
\hline
\end{tabular}




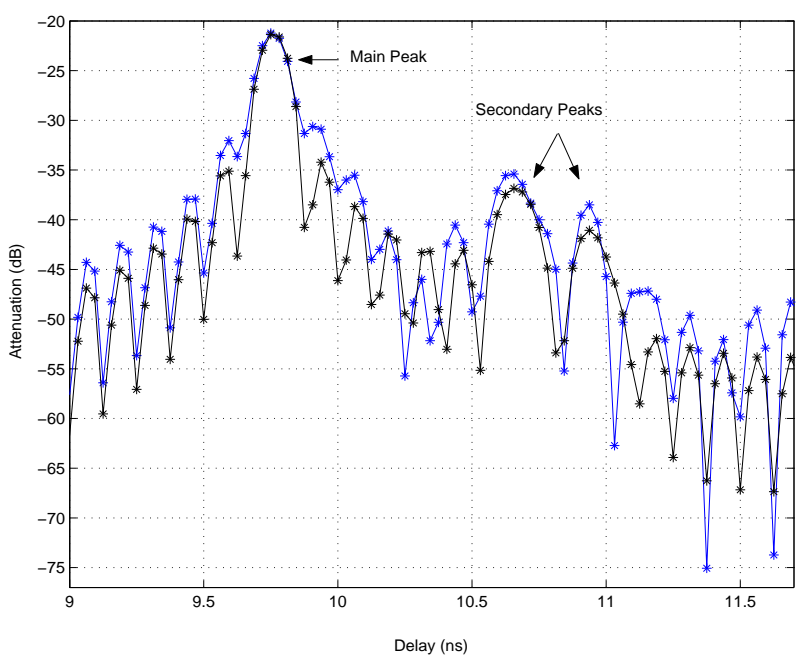

Fig. 7. Configuration 3: Time-domain analysis for hard polarization. The curves show the PL simulator prediction (black line) and the measurements (blue line).

\section{B. Transmitter and receiver at grazing aspects of incidence and observation}

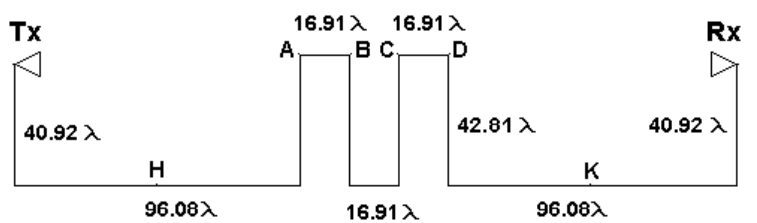

Fig. 8. Configuration 4: $T_{x}$ and $R_{x}$ at grazing incidence.

The grazing incidence configuration, shown in Figure 8, proves once again to be one of the most challenging configurations to analyze with a ray-tracing algorithm. As for the single-building configuration, the experiments were carried out by actually keeping both $T_{x}$ and $R_{x}$ below the building

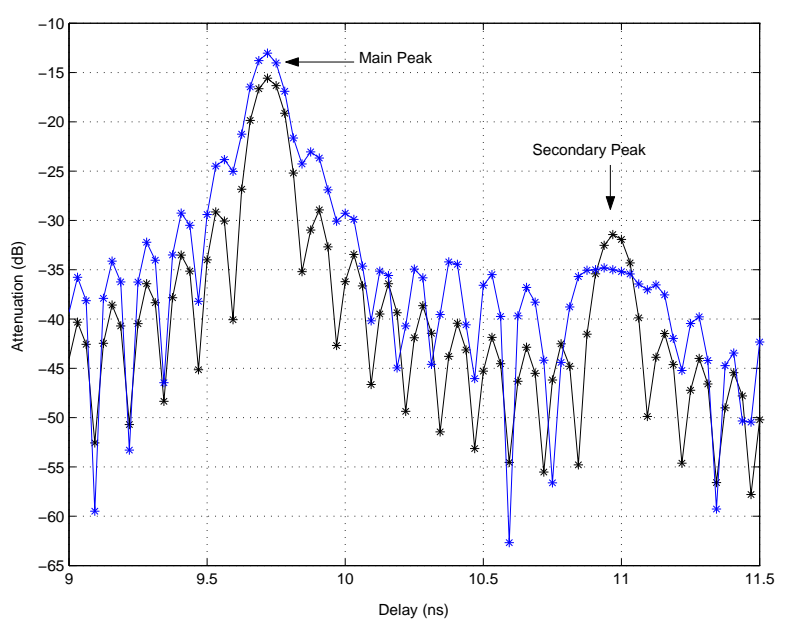

Fig. 9. Configuration 4: Time-domain analysis for hard polarization. The curves show the PL simulator prediction (black line) and the measurements (blue line). rooftop to avoid direct ray contributions.

The results for the TD analysis are shown in Figure 9. In Table $\mathrm{V}$, notice that the two secondary peaks due to trajectories 2 and 3 are again merged together in a single contribution. Both the main and the secondary peaks are predicted by the PL simulator, even though the agreement with the experiments is not as accurate as in the case of the single building profile.

TABLE V

Configuration 4: Comparison Between measured data and PREDICTIONS GIVEN BY THE PL SIMULATOR.

\begin{tabular}{|c|cc|cc|}
\hline Trj & $\begin{array}{c}\text { Measured } \\
\text { Delay (ns) }\end{array}$ & $\begin{array}{c}\text { Data } \\
\text { Attn }(\mathrm{dB})\end{array}$ & $\begin{array}{c}\text { PL } \\
\text { Delay (ns) }\end{array}$ & $\begin{array}{c}\text { Simul } \\
\text { Attn }(\mathrm{dB})\end{array}$ \\
\hline 1 & 9.720 & -13.05 & 9.724 & -15.49 \\
2 & 10.970 & -34.80 & 10.971 & -36.84 \\
3 & $"$ & $"$ & 10.973 & -38.15 \\
\hline
\end{tabular}

This behavior is attributed to the fact that the two buildings have exactly the same height (within the mechanical tolerance of the scaled profile). Under conditions of grazing aspects of incidence and observation, diffraction past the edges $A, B, C, D$ of Figure 8 requires a diffraction coefficient of order higher than the second. However, the PL simulator computes the diffraction past the two buildings by cascading the diffraction past $A, B$ with the diffraction past $C, D$. Each diffraction past a single building is computed using the diffraction coefficients described in [13].

\section{Three-Building Profile}

The three-building profile is introduced to analyze a more complex situation where there are buildings with different heights and not all surfaces are either horizontal or vertical.

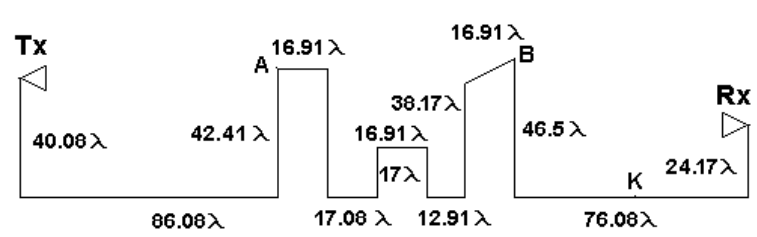

Fig. 10. Configuration 5.

Let us analyze the configuration shown in Figure 10. Though the vertices are no longer all at the same height, the $T_{x}$ height is chosen to illuminate $B$ while, at the same time, almost creating grazing incidence over the roof of the building to the left. For sake of completeness, we consider both hard and soft polarization. The three main trajectories are:
1. $T_{x} \rightarrow A \rightarrow B \rightarrow R_{x}$
2. $T_{x} \rightarrow A \rightarrow B \rightarrow K \rightarrow R_{x}$
3. $T_{x} \rightarrow H \rightarrow A \rightarrow B \rightarrow R_{x}$

The results of the TD analysis are shown in Figure 11. There is good agreement for the prediction of the main and secondary peaks, as shown in Table VI. 


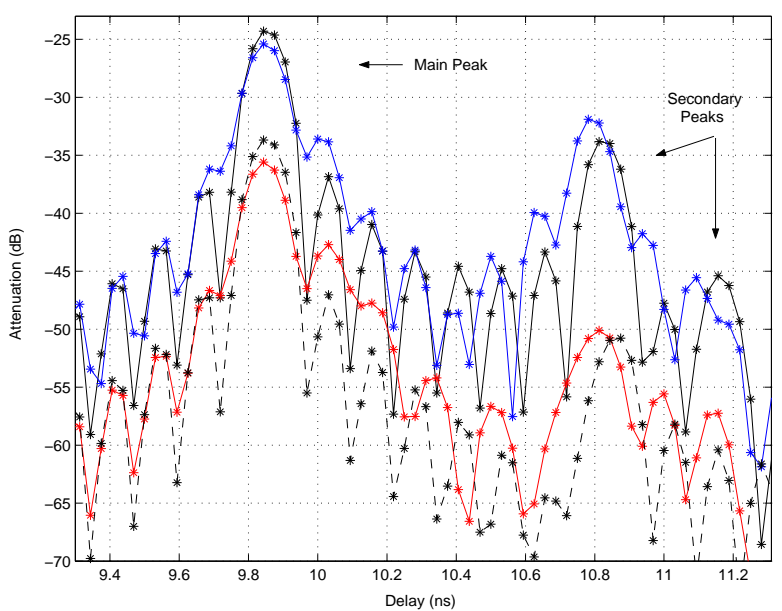

Fig. 11. Configuration 5: Time-domain analysis. The curves show the PL simulator predictions (black lines), and the measurements for soft (red line) and hard (blue line) polarization, respectively.

As expected, soft polarization provides weaker fields than hard polarization. This explains why in Figure 11 the secondary peaks for soft polarization are not so evident as in the case of hard polarization. For both polarizations, the secondary peaks are due to trajectories 2 and 3, whereas the main peaks are due to trajectory 1 . Additional trajectories are not presented here because their contributions are weaker than the background noise.

TABLE VI

Configuration 5: COMParison BetweEn MEASURED DATA AND PREDICTIONS GIVEN BY THE PL SIMULATOR.

\begin{tabular}{|c|cc|cc|}
\hline Trj & $\begin{array}{c}\text { Measured } \\
\text { Delay (ns) }\end{array}$ & $\begin{array}{c}\text { Data } \\
\text { Attn (dB) }\end{array}$ & $\begin{array}{c}\text { PL } \\
\text { Delay (ns) }\end{array}$ & $\begin{array}{c}\text { Simul } \\
\text { Attn (dB) }\end{array}$ \\
\hline \multicolumn{5}{|c|}{ Hard polarization } \\
\hline 1 & 9.875 & -25.40 & 9.850 & -24.29 \\
2 & 10.785 & -31.90 & 10.832 & -33.76 \\
3 & 10.110 & -45.55 & 11.179 & -45.39 \\
\hline \multicolumn{5}{|c|}{ Soft polarization } \\
\hline 1 & 9.845 & -35.55 & 9.850 & -33.68 \\
2 & 10.815 & -50.10 & 10.832 & -50.76 \\
3 & 11.150 & -57.25 & 11.179 & -60.40 \\
\hline
\end{tabular}

The last configuration, shown in Figure 12, presents the same profile, but with the tallest building on the transmitter side. For this configuration, the main trajectories

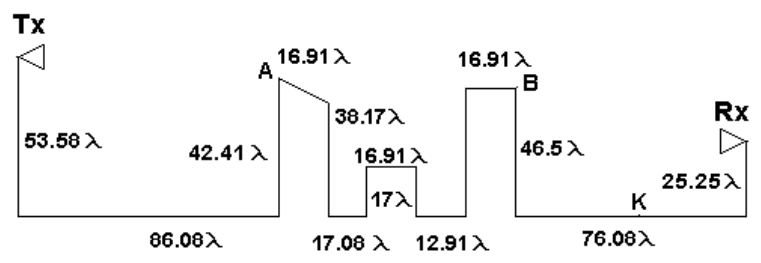

Fig. 12. Configuration 6.
TABLE VII

Configuration 6: Comparison between measured data and PREDiCtions Given By the PL Simulator. HaRd Polarization.

\begin{tabular}{|c|cc|cc|}
\hline & Measured & Data & PL & Simul \\
Trj & Delay (ns) & Attn (dB) & Delay (ns) & Attn (dB) \\
\hline \multicolumn{5}{|c|}{ Hard polarization } \\
\hline 1 & 9.720 & -13.05 & 9.724 & -15.49 \\
2 & 10.970 & -34.80 & 10.971 & -36.84 \\
\hline \multicolumn{5}{|c|}{ Soft polarization } \\
\hline 1 & 9.720 & -13.05 & 9.724 & -15.49 \\
2 & 10.970 & -34.80 & 10.971 & -36.84 \\
\hline
\end{tabular}

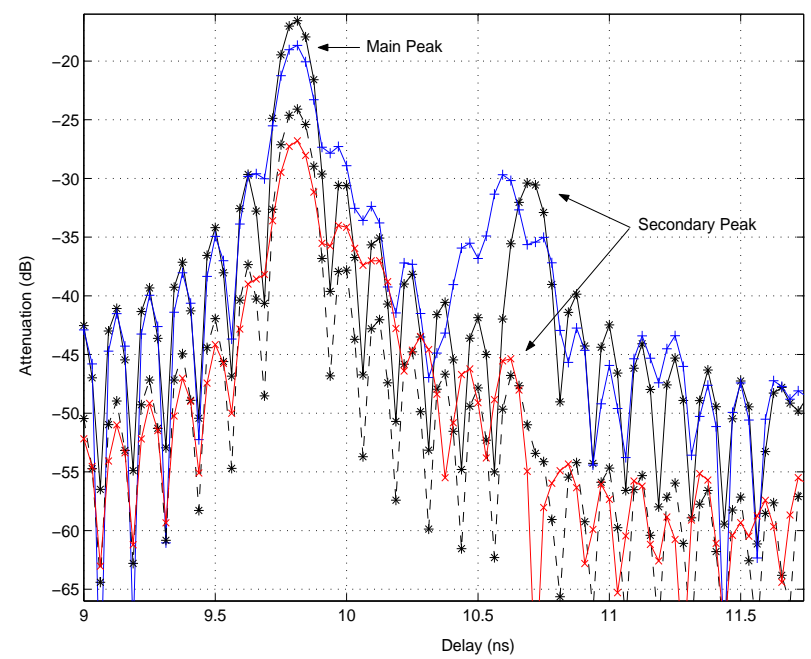

Fig. 13. Configuration 6: Time-domain analysis. The curves show the PL simulator prediction (black lines), and the measurements for soft (red line) and hard (blue line) polarization, respectively.

are:

1. $T_{x} \rightarrow A \rightarrow B \rightarrow R_{x}$

2. $T_{x} \rightarrow A \rightarrow B \rightarrow K \rightarrow R_{x}$

The results for soft and hard polarization are shown in Figure 13 as well as in Table VII. Again, there is good agreement with the theoretical results.

\section{Discussion of the Results}

The predictions obtained with the PL simulator for the single-building profile are in very good agreement with the measurements for all the configurations that were examined. The experiments with the two- and three-building profiles were focused on locations of the $T_{x}$ that cause grazing incidence and on $R_{x}$ at a short distance from the ground. This choice was made in order to better simulate the real world where it is common to have the $T_{x}$ positioned higher than the $R_{x}$, whereas the opposite hardly ever happens.

The experiment results confirm that for all these configurations the contribution of the $T_{x}$-side reflected ray is generally not negligible, as it is often comparable in amplitude with the $R_{x}$-side reflected ray. Simplified models usually account only for what happens on the $R_{x}$-side, without 
considering the $T_{x}$-side at all. This approximation can be considered acceptable when the base station is higher than the surrounding obstacles, but is definitely too rough in all the other cases.

Most of the experiments concerned grazing incidence conditions, which usually are critical for ray-tracing techniques, because of interaction with the first Fresnel zones. Nevertheless the use of the PL simulator gives results which are in good agreement with the experimental analysis.

\section{Conclusions}

This article presented an experimental study of propagation past scaled building models focusing on the different contributions due to multipath propagation. This study has been carried out to further estimate the accuracy of a two-dimensional ray tracing model for propagation in urban environment described in [1] [2] [3]. In particular, this research has isolated the components of the received signal that are most important according to the configuration of the examined profile with respect to the $T_{x}$ and $R_{x}$.

The limitations of this analysis are mainly due to the fact that it was performed on metallic models. On the other hand, the advantages of carrying out such a set of measurements in an anechoic chamber are evident, as contrasted with field measurements that are influenced by a series of factors, most of which are not easily quantifiable. It would be difficult with field measurements to determine the causes of any disagreement with the predictions. To the best of the authors' knowledge, this is the first time that a study of propagation over buildings is performed in an anechoic chamber. In literature there is only a single paper on measurements on scaled buildings [15], which however are not performed in an anechoic chamber, and that paper addresses horizontal propagation around buildings instead of over buildings.

\section{ACKNOWLEDGMENTS}

The authors are indebted to Dr. Thomas D. Monte for his technical assistance and for providing the sector antennas, and are thankful to the National Science Foundation which supported this research under grant ECS-9979413. The authors acknowledge the suggestions of Prof. Rashid Ansari for the signal processing part of the analysis, and thank Art Sawczuk, Walter Schindler and Marian Balaban of the UIC Machine Shop for manufacturing the scaled buildings and related items. Finally, they thank Giuseppe D'Elia for his assistance in the measurements, and the Reviewers for their helpful suggestions.

\section{REFERENCES}

[1] Erricolo, D. and P. L. E. Uslenghi, "Two-dimensional simulator for propagation in urban environments," IEEE Trans. Veh. Technol., Vol. 50, no. 4, pp.1158-1168, Jul 2001.

[2] Erricolo, D., Wireless Communications in an Urban Enviroment, Ph.D. thesis, University of Illinois at Chicago, Chicago, IL, USA, 1998.

[3] Erricolo, D., and P. L. E. Uslenghi, "Two dimensional ray tracing simulator for radiowave propagation in urban areas with arbitrary building shape and terrain profile," in National Radio Science Meeting, Atlanta, GA, USA, Jun 1998.
[4] Erricolo, D., and P. L. E. Uslenghi, "Comparison between raytracing approach and empirical models for propagation in urban environments.," IEEE Trans. Antennas Propagat., this issue.

[5] Erricolo, D., G. D'Elia, and P. L. E. Uslenghi, "Measurements on scaled models of urban environments and comparisons with raytracing propagation simulation." IEEE Trans. Antennas Propagat., this issue.

[6] Crovella, U. G., G. D'Elia, and D. Erricolo, "Measurements in anechoic chamber to validate EM propagation models. A tutorial overview" in National Radio Science Meeting, Boulder, CO, USA, Jan 2002.

[7] Crovella, U. G., G. D'Elia, D. Erricolo, and P. L. E. Uslenghi, "Comparison between measurements on a scaled model and a ray-tracing method for propagation in urban environments" in National Radio Science Meeting, Boulder, CO, USA, Jan 2001.

[8] Crovella, U. G., "Analysis of measurements on urban models in anechoic chamber and comparisons with propagation predictions" M.S. thesis, University of Illinois at Chicago, 2001.

[9] Erricolo, D., U. G. Crovella, and P. L. E. Uslenghi, "Timedomain measurements for path-loss prediction on a scaled model of an urban environment" in IEEE-APS/URSI Int. Symposium, Boston, MA, Jul 2001.

[10] Crovella, U. G, D. Erricolo, and P. L. E. Uslenghi, "Analysis of measurements on urban models in anechoic chamber and comparisons with propagation predictions" in Proceedings of the International conference on Electromagnetics in Advanced Applications (ICEAA), Turin, Italy, Sep 2001.

[11] HP8510C Network Analyzer. Operating and Programming Manual. Edition 2, HP Part No. 08510-90281, Hewlett Packard, 1994.

[12] Proakis, J. G., D. G. Manolakis, Digital signal processing: principles, algorithms, and applications. Englewood Cliffs, NJ: Prentice Hall, 1996.

[13] Albani, M., F. Capolino, S. Maci, and R. Tiberio, "Diffraction at a thick screen including corrugations on the top face," IEEE Trans. Antennas Propagat., vol. 45, no. 2, pp. 277-283, Feb 1997.

[14] Erricolo, D., and P. L. E. Uslenghi, "Knife edge versus double wedge modeling of buildings for ray tracing propagation methods in urban areas," in National Radio Science Meeting, Boulder, CO, USA, Jan 1998.

[15] Brown, P. G., and C. C. Constantinou, "Investigations on the prediction of radiowave propagation in urban microcell environment using ray-tracing methods," IEE Proc Microw Antennas Propagat, vol. 143, no. 1, pp. 36-42, Feb 1996.

Danilo Erricolo for a biography see [5].

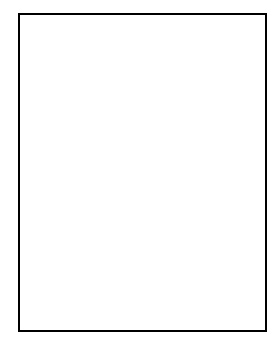

Umberto G. Crovella (S'01) received the M.S. degree in Electrical Engineering and Computer Science from the University of Illinois at Chicago and the Laurea Degree of Doctor in Electronics Engineering from the Politecnico di Torino, Turin, Italy both in 2001

He currently is a Graduate Research Assistant in the Department of Electrical and Computer Engineering, University of Illinois at Chicago. His research interests include electromagnetic communication systems. scattering, antenna measurements and wireless

Piergiorgio L.E. Uslenghi for a biography see [5]. 